\title{
Evolution of Three Parent Genes and Their Retrogene Copies in Drosophila Species
}

\author{
Ryan S. O'Neill and Denise V. Clark \\ Department of Biology, University of New Brunswick, 10 Bailey Drive, Fredericton, NB, Canada E3B $5 A 3$ \\ Correspondence should be addressed to Denise V. Clark; clarkd@unb.ca
}

Received 28 February 2013; Accepted 8 May 2013

Academic Editor: Shozo Yokoyama

Copyright (C) 2013 R. S. O’Neill and D. V. Clark. This is an open access article distributed under the Creative Commons Attribution License, which permits unrestricted use, distribution, and reproduction in any medium, provided the original work is properly cited.

\begin{abstract}
Retrogenes form a class of gene duplicate lacking the regulatory sequences found outside of the mRNA-coding regions of the parent gene. It is not clear how a retrogene's lack of parental regulatory sequences affects the evolution of the gene pair. To explore the evolution of parent genes and retrogenes, we investigated three such gene pairs in the family Drosophilidae; in Drosophila melanogaster, these gene pairs are CG8331 and CG4960, CG17734 and CG11825, and Sep2 and Sep5. We investigated the embryonic expression patterns of these gene pairs across multiple Drosophila species. Expression patterns of the parent genes and their single copy orthologs are relatively conserved across species, whether or not a species has a retrogene copy, although there is some variation in CG8331 and CG17734. In contrast, expression patterns of the retrogene orthologs have diversified. We used the genome sequences of 20 Drosophila species to investigate coding sequence evolution. The coding sequences of the three gene pairs appear to be evolving predominantly under negative selection; however, the parent genes and retrogenes show some distinct differences in amino acid sequence. Therefore, in general, retrogene expression patterns and coding sequences are distinct compared to their parents and, in some cases, retrogene expression patterns diversify.
\end{abstract}

\section{Introduction}

Gene duplication plays a major role in evolution by expanding gene families and facilitating the diversification of gene function. Following duplication, gene copies can diverge in function. Retroduplication occurs when mRNA from a parent gene is reverse-transcribed and inserted into the genome, producing a new retrogene copy that lacks the regulatory elements and introns of the parent $[1,2]$. The lack of parental regulatory elements in a new retrogene is often associated with a lack of function and pseudogenization; however, those retrogenes that are transcribed presumably lack the expression pattern of their parents and may therefore acquire novel functions [3].

Genome-wide studies have provided insights into the evolutionary outcome of this initial asymmetry between parent gene and retrogene regulatory elements and expression patterns, including several studies focusing on proteincoding parent genes and retrogenes in Drosophila. Expression data from Drosophila melanogaster show that retrogenes tend to be expressed at a lower level and in fewer tissues than their parents, with the exception that retrogenes tend to be more represented in testes than parent genes $[4,5]$. The mean expression levels of retrogenes are not significantly different from all genes in D. melanogaster [5], suggesting that the loss of parental regulatory elements does not limit the expression of functional retrogenes. An investigation of retrogene regulatory elements in $D$. melanogaster by Bai et al. [6] indicated that retrogenes do not typically acquire regulatory elements from their parent genes. Cis-regulatory element prediction has been applied to retrogenes in $D$. melanogaster, but many of these putative elements are not conserved in other Drosophila species [7]. It is unclear whether or not the expression patterns of a parent gene and retrogene are conserved across species. Conservation of a retrogene's expression pattern would indicate an early establishment of function, whereas a lack of conservation would suggest diversification of function. Also, if a parent gene's expression pattern is conserved across species, regardless of whether or not the retrogene is present, then this 
would suggest that the retrogene has evolved a novel function rather than taking over part of the parent gene's function via subfunctionalization [8].

To gain insight into the evolution of retrogene expression, we examined three gene pairs that were previously identified in D. melanogaster, each consisting of a parent gene and retrogene $[4,5]$. These $D$. melanogaster parent genes are CG8331, CG17734, and Sep2. The retrogene copies of these D. melanogaster parent genes are CG4960, CG11825, and Sep5, respectively. These gene names will be used herein for the orthologous genes in other Drosophila species. In each case, one copy is found on chromosome 2 and the other is found on chromosome 3 of $D$. melanogaster, which will facilitate genetic crosses and strain construction for future work. We chose autosomal gene pairs, rather than autosomal retrogenes with X-linked parents, in order to study diversification of gene duplicates more generally. It is thought that some of these latter duplicates were retained because they evolved male germline-specific functions that were not possible in the parent gene due to meiotic sex chromosome inactivation during spermatogenesis [9-12].

CG8331 and CG4960 are homologs of REEP5/6 (receptor accessory protein) in non-Arthropod Metazoa [13] and of Yoplp in Saccharomyces cerevisiae, an accessory protein to the Yipl p Rab GTPase [14]. CG17734 and CG11825 encode hypoxia-induced gene domain (HIGD) homologs, originally identified as an upregulated gene during hypoxia [15] and later as a mitochondrial inner membrane protein involved with cell survival under stress [16]. Sep2 and Sep5 are members of the septin family of proteins [17]. Septin function is associated with plasma membrane and cortical cytoskeleton [18]. In D. melanogaster, septins are involved in many processes, including cytokinesis [19].

We searched the genomes of sequenced Drosophila species for the presence or absence of these three gene pairs. These results allowed us to infer where in the phylogeny the retroduplication occurred. Assuming the three retroduplications occurred on the most recent branch of the Drosophila phylogeny leading to retrogene-containing descendants, then the three retrogenes arose between 12.8-35.6 (CG4960), 35.6-41.3 (CG11825), and 62.2-62.9 (Sep5) million years ago [20]. Thus, for each case, there are Drosophila species with sequenced genomes that do not have the retroduplication [21]. Comparisons between parent genes and their singlecopy orthologs should reveal how the parent gene function has changed following retroduplication [22].

Here we show that the orthologs of these parent genes and retrogenes are transcribed in 10 Drosophila species, indicating that they have not become pseudogenes. Embryonic expression patterns of parent genes, retrogenes, and singletons were determined by in situ hybridization. We find that the embryonic expression patterns of the parent gene Sep2 are conserved across the species investigated, whereas CG17734 and CG8331 have some variation among species that do not correlate with retrogene presence or absence. The three retrogenes show evidence of expression pattern diversification among species. The coding sequences of the three gene pairs appear to be evolving predominantly under negative selection, with very little evidence of positive selection; however, the parent genes and retrogenes do show distinct differences in amino acid sequence that suggest functional diversification worthy of further exploration.

\section{Materials and Methods}

2.1. Drosophila Strains. The Drosophila species strains used to generate the 12 Drosophila genomes [21, 26, 27] were used in this study. D. melanogaster strain $y$; Gr22b Gr22d cn CG33964 ${ }^{R 4.2}$ bw sp; LysC MstProx GstD5 Rh6 was obtained from Bloomington Drosophila Stock Center at Indiana University. D. pseudoobscura 14011-0121.94, D. ananassae 14024-0371.13, D. erecta 14021-0224.01, D. mojavensis 15081-1352.22, D. sechellia C 14021-0248.25, D. simulans w501 14021-0251.195, D. virilis 15010-1051.87, D. willistoni 140300811.24, and D. yakuba 14021-0261.01 were obtained from the Drosophila Species Center at UC San Diego, CA, USA.

2.2. Sequences. Coding and amino acid sequences of all transcripts for CG8331, CG4960, CG17734, CG11825, Sep2, and Sep5 in D. melanogaster were obtained from FlyBase release FB2012_02 [28, 29]. To identify the parent gene transcript that likely gave rise to the retrogene, all pairwise alignments of coding sequences derived from alternative transcripts in $D$. melanogaster were generated using the Needleman-Wunsch algorithm [30]. The coding sequences of these homologous transcripts were used for further sequence analyses.

Amino acid and coding sequences of the orthologs in other sequenced Drosophila species were obtained from FlyBase [28] using the coding sequences of the D. melanogaster genes as BLAST [31] queries. If a BLAST search resulted in a predicted gene model, then coding and protein sequences were obtained from that gene model; if no gene model existed for a particular search result, then coding and protein sequences were predicted using GeneWise [32]. If no ortholog was found in a particular species, we determined whether its absence was due to a deletion or an absence of genomic sequence data by performing BLAST searches, using the D. melanogaster genomic sequences flanking these genes as queries. In some cases, no BLAST hit corresponded to $>100$ kilo bases of sequence containing the gene in $D$. melanogaster, suggesting that the gene may be absent due to a gap in that species genome assembly. We chose to include sequences from all available sequenced Drosophila species to increase the power of our analyses for detecting selection acting on gene pairs. Accession numbers of the genes and genomes used are listed in Supplementary File 1 in Supplementary Material available online at http://dx.doi.org/10.1155/2013/693085.

Codon alignments of each gene pair were constructed by aligning protein sequences using Clustal Omega [33] with default settings, reverse-translating the protein alignment into a codon alignment with PAL2NAL [34], and then checking the alignments and removing codons that contained gaps or that were ambiguously aligned in some species [35]. For each codon alignment, MEGA5 [36] was used to determine the best model of sequence evolution and then construct a phylogenetic tree using maximum likelihood. Trees were visualized using iTOL $[37,38]$. 
2.3. Quantitative Reverse Transcriptase PCR ( $q R T-P C R)$. Total RNA was extracted from one pool of ten 2-4-day-old males or females using TRIzol (Invitrogen). RNA was DNase treated using RQ1 RNase-Free DNase (Promega). cDNA synthesis was performed using the reverse transcriptase (RT) enzyme mix included in the SuperScript III Platinum TwoStep qRT-PCR Kit (Invitrogen). Gene-specific primers were designed using Primer3 [39] for orthologous gene pairs and RpL32 in 10 Drosophila species (Supplemental File 1). Each qRT-PCR was performed with three technical replicates and one RT control using the Corbett Rotor-Gene 6000. Cross threshold (CT) values and PCR efficiencies were determined using LinReg, version 11.1 [40]. CT values of the technical replicates were averaged and efficiency corrected to $100 \%$ using the formula $\mathrm{CT}_{100 \% \text { efficiency }}=\mathrm{CT} *[\log (1+$ efficiency)/ $\log (2)]$ [41]. Relative quantification (RQ) of genes was determined using the formula $R Q=2^{\text {(CTgeneA-CTgeneB) }}$ [41]. To compare transcripts within RNA samples, the ratios of parent gene to retrogene transcripts were calculated.

2.4. In Situ Hybridization. For each gene pair, we chose to investigate expression patterns in a subset of Drosophila species spread throughout the phylogeny. In each case, we included at least two species without the retrogene. DNA templates for sense and antisense RNA probe synthesis were amplified from cDNA using gene-specific primers (Supplemental File 1). A T7 RNA polymerase promoter sequence (TAATACGACTCACTATAG + A/G) was added to the $5^{\prime}$ end of either the forward primer to synthesize sense probes or the reverse primer to synthesize antisense probes. Templates were gel-purified, using QIAEX II Gel Extraction Kit (Qiagen), and sequenced using Sanger sequencing. Sense and antisense digoxigenin- (DIG-) labeled RNA probes were synthesized from purified probe templates using T7 RNA polymerase and DIG RNA labeling mix (Roche Applied Science). Probes were DNase-treated, precipitated overnight, and resuspended in DEPC-treated distilled water. Probe yield was estimated by spotting dilutions of probe, along with DIG-labeled control RNA (Boehringer Mannheim), onto positively charged nitrocellulose, incubating with Anti-DIG-AP antibody (Roche) and detecting using AP Buffer $(100 \mathrm{mM} \mathrm{NaCl}, 50 \mathrm{mM} \mathrm{MgCl}$, $100 \mathrm{mM}$ TrisCl pH 9.5, and 0.1\% Tween-20) with NBT and BCIP (Roche).

Zero- to five-day-old flies were aged in bottles with food and live yeast paste for 3 days. Embryos were collected in Embryo Collection Cages (Genesee) on grape agar plates with live yeast paste for 9 or 18 hours (18 or 36 hours for $D$. virilis). Eggs were dechorionated with $50 \%$ bleach, then fixed in $4 \%$ formaldehyde in $1 \mathrm{x}$ PBS and heptane for 20 minutes, shaken in methanol, and stored at $-20^{\circ} \mathrm{C}$. In situ hybridization was performed according to Tautz and Pheifle [42], with modifications from [43, 44]. Embryos were warmed to room temperature and rehydrated by washing in 3:1 methanol: PBST, 1:3 methanol: PBST, and then PBST (1x PBS, 0.1\% Tween-20). Embryos were fixed with $4 \%$ formaldehyde in PBST, digested for 4 minutes with $25 \mu \mathrm{g} / \mathrm{mL}$ proteinase $\mathrm{K}$ (Merck), washed for 2 minutes with $2 \mathrm{mg} / \mathrm{mL}$ glycine in PBST, and fixed again with $4 \%$ formaldehyde in
PBST. Embryos were washed with 50\% hybridization solution (50\% formamide, 5x SSC, $100 \mu \mathrm{g} / \mathrm{mL}$ heparin, $100 \mu \mathrm{g} / \mathrm{mL}$ sonicated salmon sperm DNA, and $0.1 \%$ Tween-20) in PBST for 5 minutes and then incubated for 2 hours at $56^{\circ} \mathrm{C}$ with hybridization solution that was boiled for 5 minutes and then chilled on ice for 5 minutes. Probe was added to hybridization solution at about $50 \mathrm{ng} / \mathrm{mL}$, incubated at $80^{\circ} \mathrm{C}$ for 3 minutes, and chilled on ice for 5 minutes. Embryos were hybridized with this probe solution for 16 hours at $56^{\circ} \mathrm{C}$, washed in progressive dilutions of hybridization solution and PBST, then washed with PBST. Embryos were cooled to room temperature and incubated with a 1:2000 dilution of antidigoxigenin-AP antibody in PbT (1x PBS, 0.1\% Triton X100, and $0.2 \%$ bovine serum albumin). Embryos were washed with PBST and then AP buffer, before staining with AP buffer containing NBT and BCIP. The color reaction was stopped by washing several times in PBST, then ethanol, then PBST, and the embryos were placed in a solution of $70 \%$ glycerol in PBS and stored at $4^{\circ} \mathrm{C}$. Embryos were mounted on slides and viewed under differential interface contrast using the Leica DM RXA2 microscope. Individual embryos were staged according to Campos-Ortega and Hartenstein [24] and assessed for reproducible staining patterns. Photomicrographs were captured using a Leica DC500 camera and ThumbsPlus 4.0 software and were rotated and cropped using Fiji software.

2.5. Analyses of Coding Sequences. Structural features of the proteins encoded by the genes in this study were predicted using TMHMM v. 2.0 [45] for transmembrane helices and Multicoil2 [46] for coiled coils.

Nonsynonymous and synonymous substitution rates $(\mathrm{dN}$ and $\mathrm{dS}$, resp.) and the ratio of $\mathrm{dN} / \mathrm{dS}(\omega)$ can be used to infer selection: $\omega<1$ implies purifying selection, $\omega=1$ implies neutral evolution, and $\omega>1$ implies positive selection. We used several methods to test for selection acting on the gene pairs in this study. Codon alignments, with ambiguities and gaps removed, were used in each test (Supplemental File 6).

To test for positive selection acting specifically on either retrogenes or parent genes, we performed the branch-site test [47], implemented in the CODEML program of the PAML package version 4 [48]. The branch-site test considers variation in $\omega$ across branches and codons of a phylogenetic tree to determine whether the pattern of selection on a specified lineage (foreground) is significantly different from that on the rest of the tree (background). A maximum likelihood approach is used to compare different models and infer positive selection acting on the specified lineage. The null model allows only purifying or neutral selection. The alternative model constrains codons in the background to purifying or neutral selection, while allowing codons in the foreground to undergo purifying, neutral, or positive selection. If a likelihood ratio test (LRT) of the alternative versus the null model is significant, then positive selection on the foreground branches is inferred. Significance of the LRT is assessed using a chi-squared distribution with 1 degree of freedom. In cases where positive selection is inferred, the Bayes Empirical Bayes (BEB) method [49] is used to estimate 
which codons evolved under positive selection. For each PAML analysis, we submitted a custom tree that constrained the relationships between genes to the relationships between species on the Drosophila phylogeny [21, 23], thus assuming that each retrotransposition occurred on the most recent branch leading to the clade containing all species with the retrogene. We designated the branch giving rise to either the retrogene or the parent gene (excluding the singletons) as the foreground branch to test for positive selection on that clade.

We used the Datamonkey server $[50,51]$ to perform additional selection analyses. Substitution models were chosen for each gene pair using the model selection tool. Neighbourjoining trees generated on the Datamonkey server were used for the analyses. FEL [52] was used to detect sites evolving under negative selection in both the parent and retrogene. MEME [53] was used to detect sites that underwent episodic diversifying selection in a subset of lineages. We used a $P$ value cutoff of 0.05 for both FEL and MEME analyses, which should be conservative based on simulations $[52,53]$.

Visual inspection of the multiple sequence alignments for each gene pair revealed some amino acid sites that were obviously different between the parent gene and retrogene, particularly in Sep2 and Sep5, but which were not identified by the selection analyses listed above. Therefore, we manually identified sites with $\geq 80 \%$ amino acid conservation across parent genes (including singletons), $\geq 80 \%$ amino acid conservation across retrogenes, but which were different between the parent and retrogene.

\section{Results and Discussion}

3.1. Determination of Paralogous Coding Sequences. For each gene pair, alternative transcripts from the parent gene and retrogene in D. melanogaster were obtained from FlyBase [29], and pairwise sequence alignments of the coding regions from these transcripts were performed to identify the paralogous coding sequences. These paralogous coding sequences were used to determine the orthologous coding sequences of gene pairs in other Drosophila species. The three retroduplications are shown in Figure 1, and details of the coding sequence pairwise analyses are in Supplemental File 1.

\subsection{Identification of Orthologous Parent Genes and Retro-} genes. We used BLAST [31] to identify orthologs of CG8331, CG4960, CG17734, CG11825, Sep2, and Sep5 in the sequenced Drosophila species (Supplemental File 1). BLAST reported two copies of CG11825 in D. yakuba with 100\% nucleotide sequence identity, so only one copy was used for further analyses. BLAST also reported two copies of CG17734 in D. ficusphila; one has a frameshift mutation near the $3^{\prime}$ end and so was excluded from further sequence analyses. CG11825 appears to have been lost in $D$. erecta. Maximum-likelihood trees of each gene pair show that orthologous retrogenes form monophyletic clades, supporting a single retroduplication in each case (Figure 2).

3.3. Investigation of Parent Gene and Retrogene Expression across Species. To assess functionality of CG8331, CG4960,

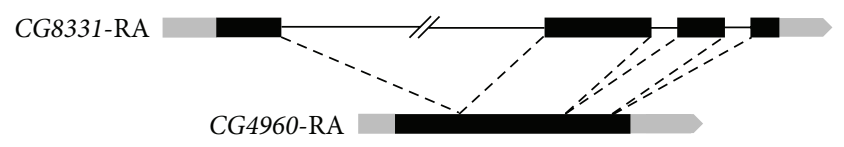

(a)

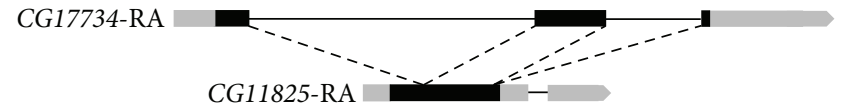

(b)

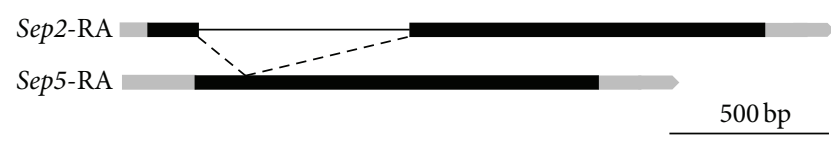

(c)

FIGURE 1: Three retroduplications in D. melanogaster. For each gene pair, coding sequences of transcripts were aligned to determine the most similar transcripts between parent gene and retrogene. Black bars indicate coding sequence, grey bars indicate untranslated regions, and thin lines indicate introns. Dashed lines show intron loss from parent gene to retrogene. Gene models were obtained from FlyBase. (a) CG4960 is a retroduplication of either CG8331RA (shown) or CG8331-RD, which encodes identical proteins. (b) CG17734-RA gave rise to CG11825. CG11825-RA is the retrogene transcript with the highest level of sequence identity to any transcript of CG17734. (c) Sep2 encodes a single transcript. Sep5 encodes 2 transcripts, Sep5-RA (shown) and Sep5-RB, which both contain identical coding sequence.

CG17734, CG11825, Sep2, and Sep5 in Drosophila species other than D. melanogaster, we confirmed their expression in adult males and females of 10 species using qRT-PCR (Supplemental Figure 5). In each species, the retrogenes showed lower expression compared to their parent genes.

To explore the evolution of parent gene and retrogene expression patterns, embryonic expression patterns of the three gene pairs were detected by in situ hybridization of antisense RNA probes in various species. Sense-probe treatments were done in parallel to assess background staining (Supplemental File 7). Unless otherwise noted, background staining was not detected. We compared our embryonic expression patterns in D. melanogaster to those generated by the Berkeley Drosophila Genome Project (BDGP) [54, 55] when possible. Data from FlyAtlas [56] and the modENCODE developmental transcriptome of D. melanogaster [57] presented on FlyBase [29] provided additional information on gene expression (summarized in Supplemental File 1), complementing the qualitative expression patterns presented here.

3.4. Parent Gene CG8331 and Retrogene CG4960. CG8331 transcript is detected in the salivary glands of late-stage embryos in all species examined (Figure 3). D. melanogaster also shows maternal transcript, which disappears by cellular blastoderm and low-level ubiquitous transcript appearing again at the end of gastrulation. D. simulans shows lowlevel ubiquitous transcript only detectable at the end of embryogenesis. D. yakuba also shows ubiquitous staining 


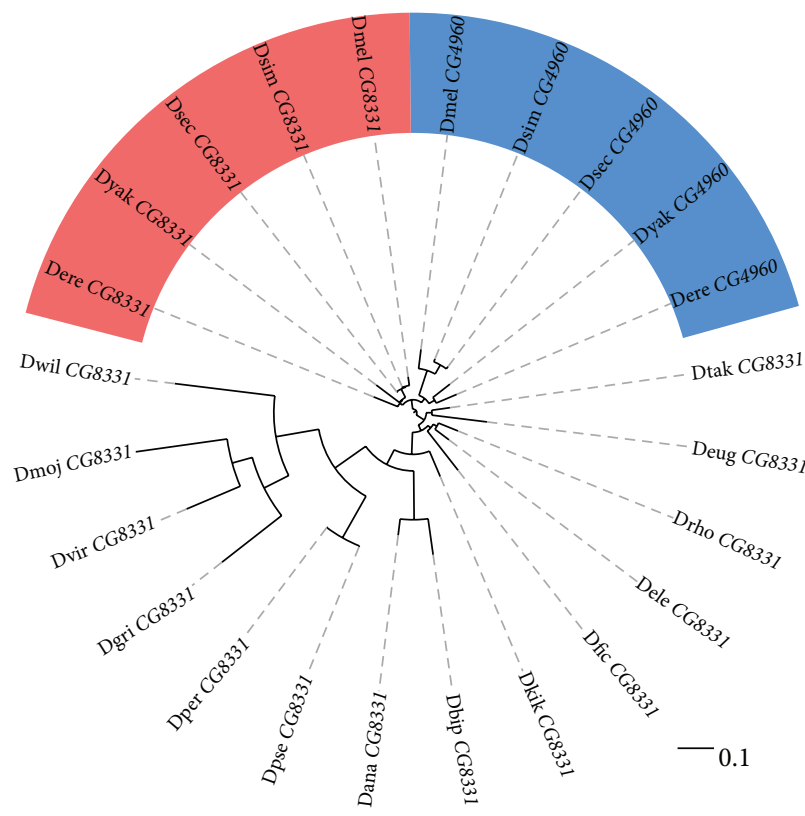

(a)

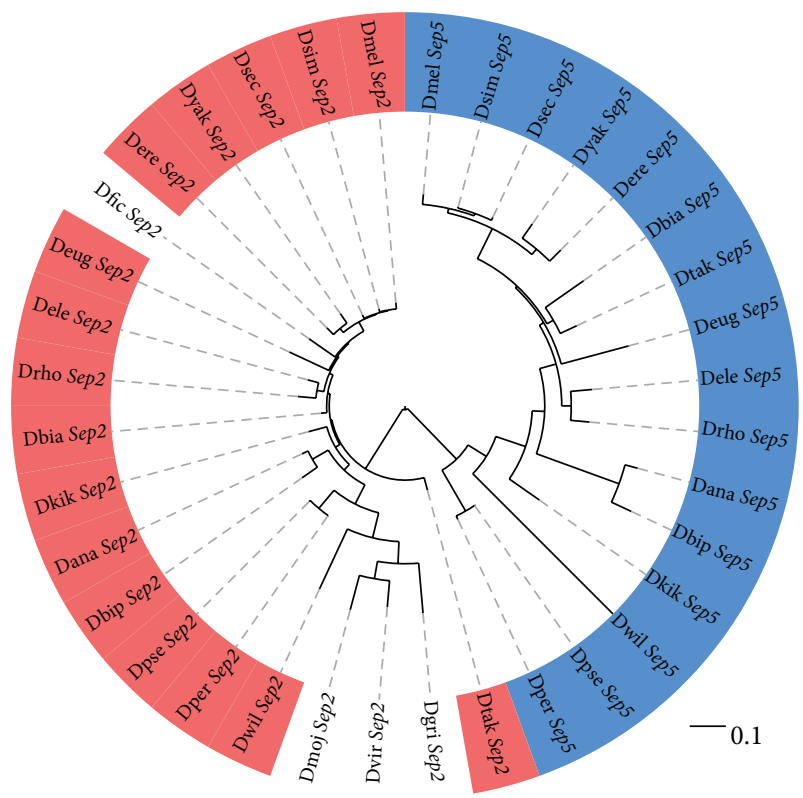

(c)

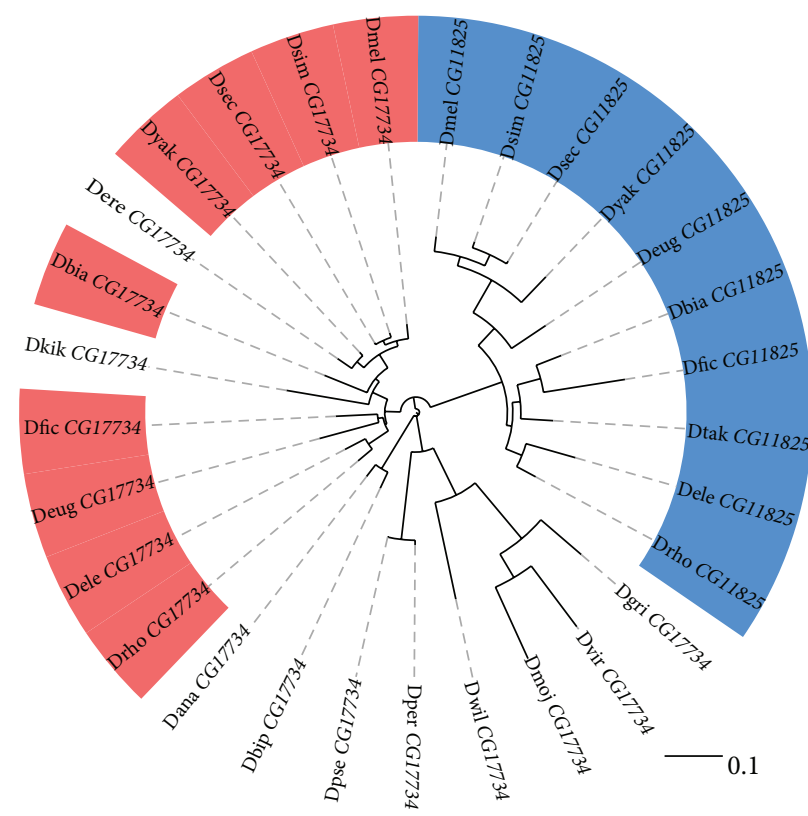

(b)

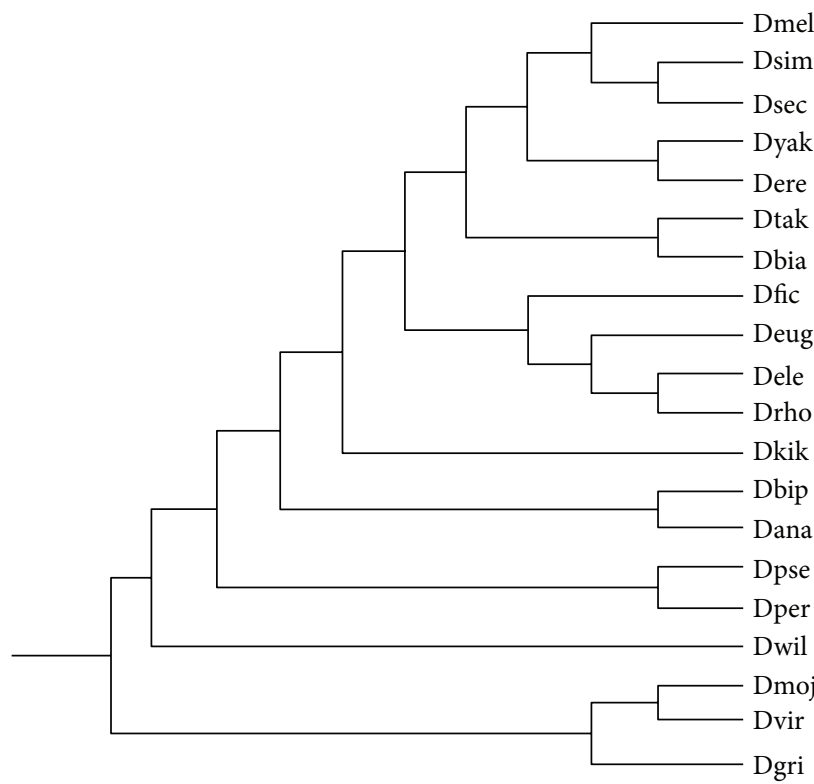

(d)

FIGURE 2: Phylogenetic analysis of three gene pairs shows that parent genes and retrogenes form independent clades. Maximum-likelihood trees were constructed from codon alignments of orthologs of each gene pair. Pink and blue highlightings correspond to parent genes and retrogenes, respectively. Trees are rooted to highlight relationships between parent genes, retrogenes, and singletons. (a) CG8331 and CG4960 (CG8331 is missing from Dbia due to a gap in the genome assembly); (b) CG17734 and CG11825 (CG17734 is missing from Dtak due to a gap in the genome assembly, and CG11825 was deleted in Dere); (c) Sep2 and Sep5 (Sep5 is missing from Dfic due to a gap in the genome assembly); (d) cladogram showing phylogeny of sequenced Drosophila species, according to Yang et al. [23]. Dmel, D. melanogaster; Dsim, D. simulans; Dsec, D. sechellia; Dyak, D. yakuba; Dere, D. erecta; Deug, D. eugracilis; Dbia, D. biarmipes; Dtak, D. takahashii; Dfic, D. ficusphila; Dele, D. elegans; Drho, D. rhopaloa; Dkik, D. kikkawai; Dbip, D. bipectinata; Dana, D. ananassae; Dpse, D. pseudoobscura; Dper, D. persimilis; Dwil, D. willistoni; Dmoj, D. mojavensis; Dvir, D. virilis; Dgri, D. grimshawi.

throughout embryonic development, although there was some background following the sense probe negative control. $D$. ananassae, D. pseudoobscura, and D. virilis also show ubiquitous staining throughout embryonic development. FlyAtlas found that CG8331 is expressed in D. melanogaster larvae and adults in all tissues investigated, with the highest level of expression in salivary glands. The modENCODE developmental transcriptome of D. melanogaster shows that CG8331 is highly maternally expressed, is reduced from cellular blastoderm through to gastrulation, and then peaks 


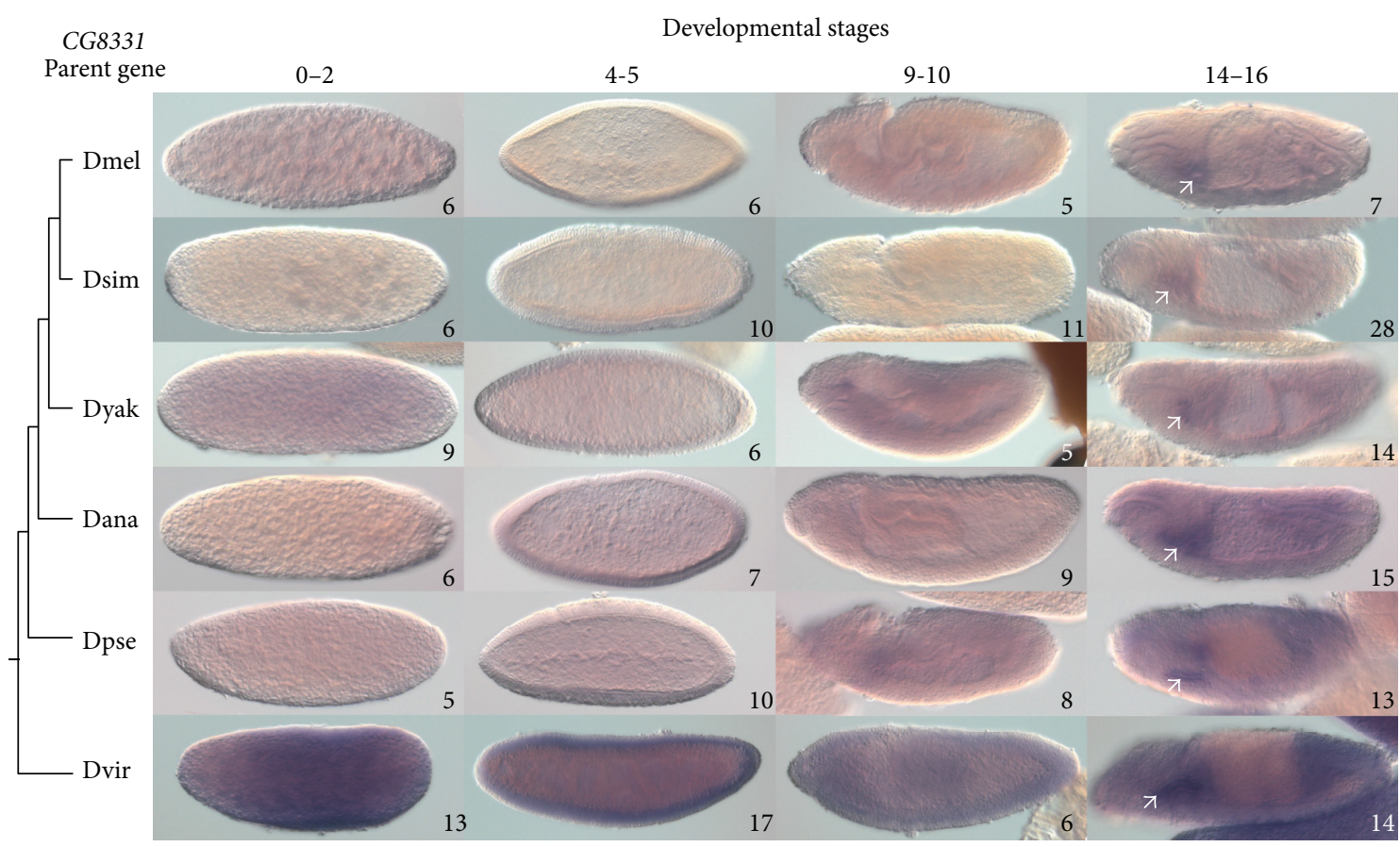

CG4960

Retrogene

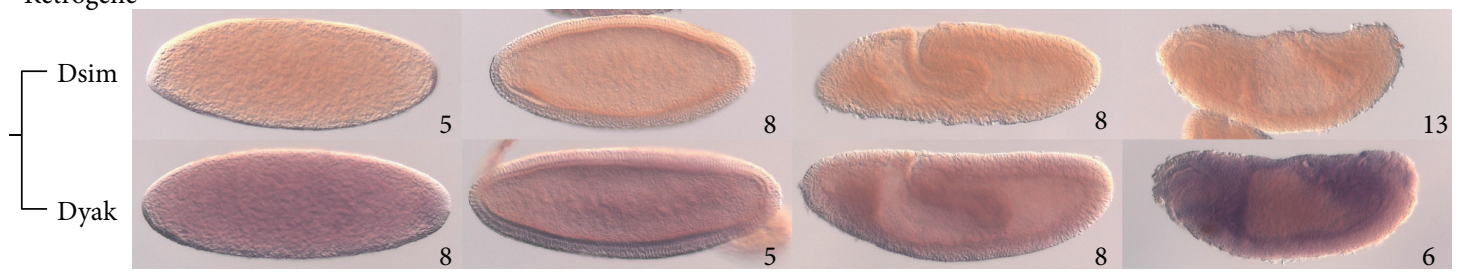

Figure 3: Expression patterns of CG8331 and CG4960. Expression of CG8331 and CG4960 was detected in Drosophila species by in situ hybridization as shown in the top and bottom panels, respectively. Purple coloration indicates presence of mRNA. Cladograms show the relationships of the species investigated. Numbers on individual panels indicate the number of embryos assessed with consistent staining patterns. White arrows point to salivary glands. Stage ranges are according to [24]: 0-2, syncytial blastoderm; 3-4, cellular blastoderm; 9-10, during germ-band elongation; 14-16, final embryonic stages. Staining during stages 0-2 indicates presence of maternal transcripts [25]. Dmel, D. melanogaster; Dsim, D. simulans; Dyak, D. yakuba; Dana, D. ananassae; Dpse, D. pseudoobscura; Dvir, D. virilis.

in expression during germ-band retraction, when the salivary glands develop [24]. These observations indicate that, except in D. simulans, CG8331 has maternal expression and ubiquitous expression through early embryogenesis, and all species showed expression in salivary glands, with a lower level of ubiquitous expression in late embryogenesis.

CG4960 was not investigated in D. melanogaster since no embryonic transcription was detected in the modENCODE developmental transcriptome. However, FlyAtlas and the modENCODE developmental transcriptome data indicate that CG4960 has adult male testis-specific expression in $D$. melanogaster. Likewise, no embryonic expression was detected in D. simulans (Figure 3). However, CG4960 is expressed ubiquitously throughout embryogenesis in $D$. yakuba, with dark staining in the midgut during later stages of embryonic development. These observations show that CG4960 has diversified in expression pattern.

Testis expression of CG4960 appears conserved across species: Zhang et al. [58] found that CG4960 is upregulated in D. yakuba males compared to females. Retrogenes are often expressed in testes in Drosophila $[4,5]$ and other species $[59,60]$. The "out of the testis" hypothesis proposes that this is a common initial expression pattern for retrogenes, which could allow a retrogene to persist and evolve new expression patterns [59]. This was not found to be a trend for Drosophila retrogenes [4], although it could apply to individual cases of retroduplication. Bai et al. [6] showed that testes expression of Drosophila retrogenes can be due to genomic position within testis-biased gene neighborhoods. modENCODE tissue expression data presented on FlyBase shows that six out of eight of the neighboring genes within 20 kilobases flanking either side of CG4960 are expressed higher in testis compared to other tissues, suggesting that CG4960 is located within a testis-biased gene neighborhood. If the embryonic expression of CG4960 in D. yakuba is a derived characteristic, it may be a good model for studying the "out of the testis" hypothesis.

3.4.1. Parent Gene CG17734 and Retrogene CG11825. Maternal expression of CG17734 was detected in all species 


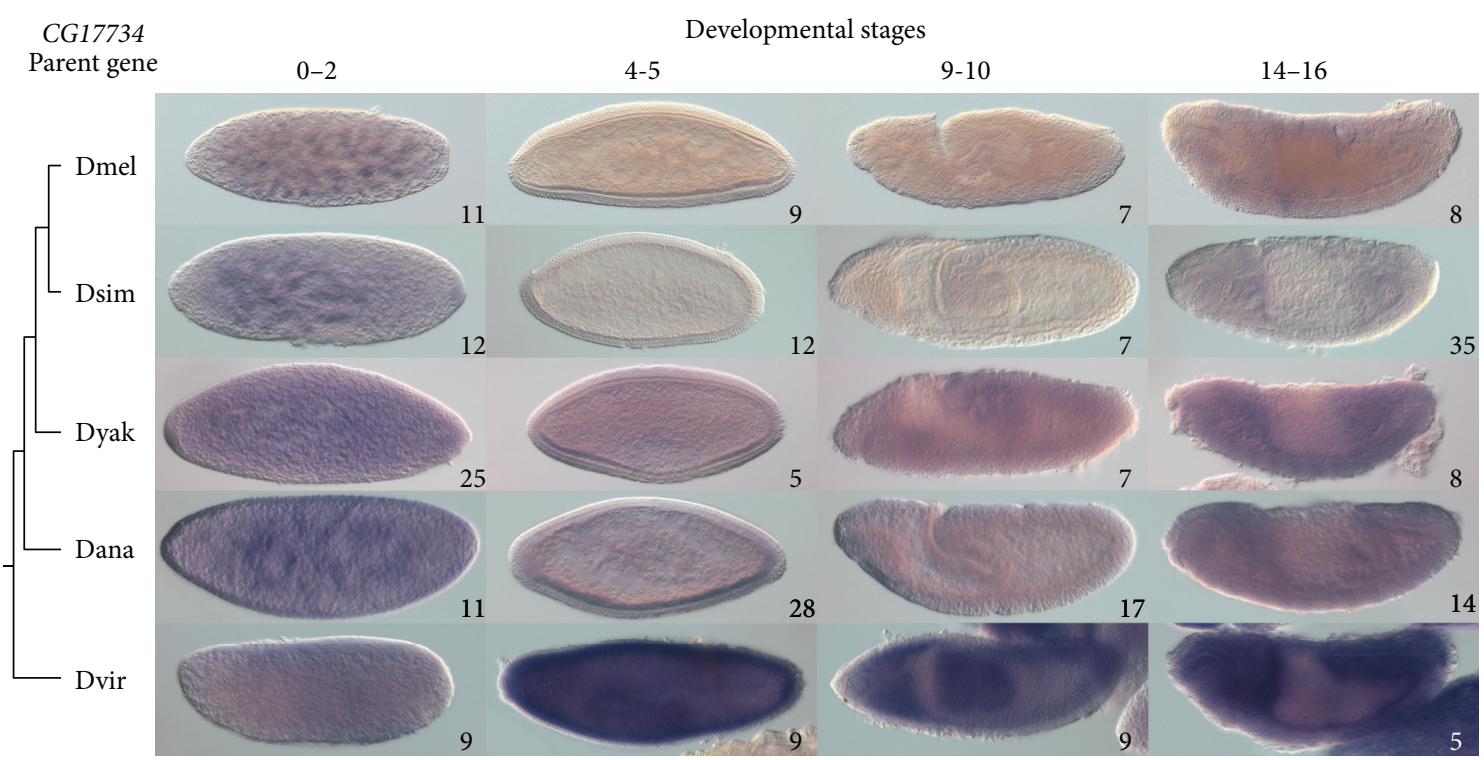

\section{CG11825}

Retrogene

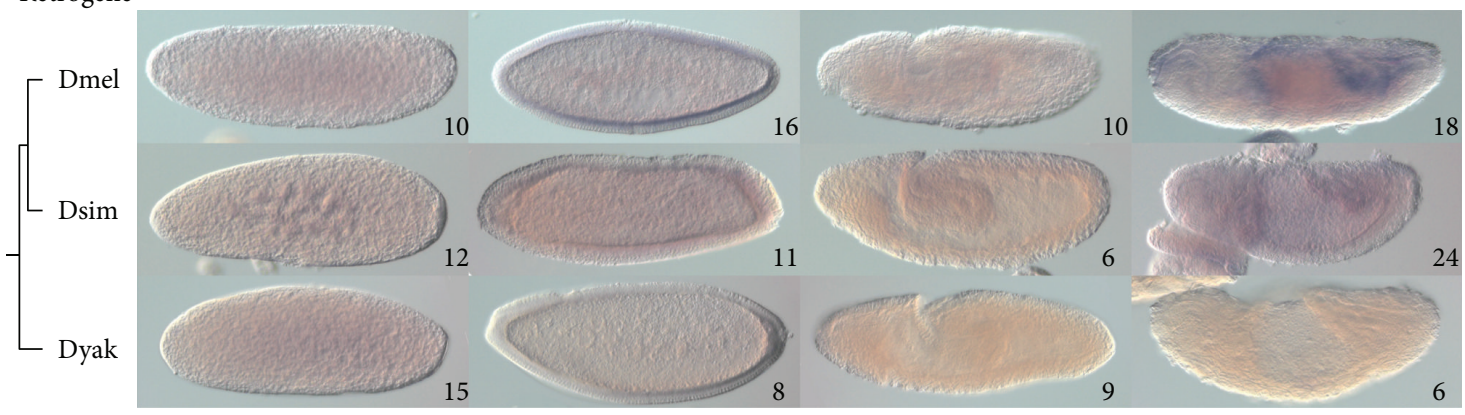

FIGURE 4: Expression patterns of CG17734 and CG11825. Expression of CG17734 and CG11825 was detected in Drosophila species by in situ hybridization as shown in the top and bottom panels, respectively. Purple coloration indicates presence of mRNA. Cladograms show the relationships of the species investigated. Numbers on individual panels indicate the number of embryos assessed with consistent staining patterns. Stage ranges are according to [24]: 0-2, syncytial blastoderm; 3-4, cellular blastoderm; 9-10, during germ-band elongation; 14-16, final embryonic stages. Staining during stages 0-2 indicates presence of maternal transcripts [25]. Dmel, D. melanogaster; Dsim, D. simulans; Dyak, D. yakuba; Dana, D. ananassae; Dvir, D. virilis.

investigated (Figure 4). In D. melanogaster, CG17734 transcript is detected weakly during syncytial blastoderm and gastrulation, and the transcript is ubiquitous during late development. The modENCODE developmental transcriptome for D. melanogaster indicates that CG17734 transcript is also present at relatively low to moderate levels from cellular blastoderm until late embryonic development. The BDGP found that, in D. melanogaster, CG17734 is expressed maternally, is rapidly degraded during cellular blastoderm, and is expressed in the developing germline during gastrulation and later stages. In D. simulans, CG17734 is also weakly expressed in the cellular blastoderm and throughout gastrulation and ubiquitously expressed during late embryogenesis. CG17734 transcript is detected ubiquitously throughout embryogenesis in D. yakuba, D. ananassae, and D. virilis, but the intensity of the staining across embryonic stages in these species does not indicate a reduction of expression from cellular blastoderm through to gastrulation. D. virilis shows more intense staining surrounding the midgut during late embryogenesis; however, our in situ hybridization protocol resulted in more intense staining of this species generally. So, CG17734 has conserved maternal expression and ubiquitous expression in late embryogenesis, but there is some variation in the intensity of expression during cellular blastoderm and gastrulation in D. melanogaster and D. simulans.

CG11825 is maternally expressed and has a low level of expression during cellular blastoderm stages in $D$. melanogaster, D. simulans, and D. yakuba (Figure 4). CG11825 is also weakly expressed throughout later embryogenesis in D. melanogaster and D. simulans, with more intense staining around the midgut and hindgut; however, it is not expressed here in D. yakuba. The modENCODE developmental transcriptome reports that CG11825 transcript is highest during the early stages of embryogenesis in D. melanogaster, but is also expressed at a moderate level throughout embryogenesis, with a second peak at age of 10-12 hours, corresponding to embryonic stages 13-15. The BDGP found that, in $D$. melanogaster, CG11825 is expressed maternally and is not 
expressed in the embryo after the cellular blastoderm stage; however, because BDGP used a probe based on the cDNA of the transcript CG11825-RA, they may have missed the other transcripts that our probe would detect. So, although expression of CG11825 in early embryogenesis is conserved across the three species, expression in later development is only present in $D$. melanogaster and D. simulans, suggesting that CG11825 has diversified in expression pattern.

Both D. melanogaster and D. simulans, compared to the other species investigated, showed expression differences in CG17734 and CG11825. These expression differences in CG17734 and CG11825 appear to be complementary in these two species: CG17734 has lower expression during cellular blastoderm and gastrulation, while CG11825 has expression from cellular blastoderm into later embryogenesis. Transcript levels of CG17734 and CG11825 in D. melanogaster, determined by modENCODE [57] (Supplemental File 1), also show complementary levels of expression over the course of embryonic development. These observations suggest subfunctionalization of expression pattern [8], which occurs when expression of one gene copy compensates for loss of expression of its paralog. However, it is difficult to make this conclusion. Transcript levels may not accurately reflect posttranscriptional gene activity, and paralogous proteins may not be functionally equivalent.

3.4.2. Parent Gene Sep2 and Retrogene Sep5. Sep2 transcript is expressed ubiquitously throughout embryonic development in the six species examined (Figure 5). In D. melanogaster and D. yakuba, there was particularly dark staining in bands of somatic muscle during the final stages of embryonic development. The BDGP found that Sep2 is expressed ubiquitously throughout embryogenesis in $D$. melanogaster, with more intense staining in the nervous system and dorsal vessel of late-stage embryos which we did not observe. The modENCODE developmental transcriptome database reports Sep2 expression throughout embryogenesis in $D$. melanogaster.

Sep5 is expressed during cellular blastoderm in all species examined (Figure 5). In D. melanogaster, Sep5 shows three relatively dark staining bands at the anterior, medial, and posterior regions of the cellular blastoderm. The BDGP found a similar pattern during cellular blastoderm and also found staining along the ventral side of the embryo at the onset of gastrulation and in the endoderm and head mesoderm during gastrulation which we did not observe. In D. willistoni, Sep5 shows darker staining at the anterior and posterior ends during cellular blastoderm. In D. yakuba and D. pseudoobscura, Sep5 shows uniform staining during the cellular blastoderm stage and is also ubiquitously expressed throughout embryogenesis. These observations suggest that Sep5 has diversified in expression pattern, while Sep 2 retained the ancestral expression pattern.

Sep2 forms a complex with the Drosophila group 2B septins, Sep1 and pnut, during embryogenesis in a 1:1:1 ratio, and these three proteins form filaments in vitro [61]. One explanation for the conservation of Sep2 expression pattern in Drosophila species embryos is that all three proteins are required for a functional complex. The BDGP determined that Sep1 is ubiquitously expressed until the final stages of embryogenesis, and the modENCODE developmental transcriptome shows that all three genes are similarly expressed during embryogenesis. Sep5 may integrate into the Sep1pnut-Sep2 complex during cellularization too [19]. Protein interaction data show that both Sep2 and Sep5 can interact with each other and with Sep1 and pnut, whereas other protein interactions are not shared [62]. Expression of the retrogene Sep5 during embryogenesis likely has functional consequences for the septin complex and may regulate how the complex interacts with other proteins or membranes.

3.5. Sequence Evolution of Three Gene Pairs. TMHMM and Multicoil2 were used to predict additional protein features of the paralogous coding sequences of each gene pair (detailed in Supplemental File 1, visually summarized in Supplemental Figures 2, 3, and 4). CG8331 and CG4960 encode proteins with two transmembrane helices, consistent with observations from the yeast homolog Yoplp [14]. The transmembrane helices are well conserved across orthologs and paralogs, except in the case of CG8331 in D. ficusphila which was predicted to have four smaller helices. CG17734 and CG11825 appear to encode proteins with two transmembrane helices, consistent with observations from the mouse homolog HIMP1 [16]. The position of the first transmembrane helix of CG17734 and CG11825 is absolutely conserved across orthologs and paralogs. The position of the second helix of CG17734 and CG11825 is well conserved, but in more distantly related orthologs of CG17734, and nearly all orthologs of CG11825, this second transmembrane domain was not scored as significant by TMHMM. Both Sep2 and Sep5 contain a coiled-coil domain toward the C-terminus; the position of this domain is conserved across orthologs; however, the coiled-coil domain of Sep5 is predicted not to extend as far as that of Sep2.

We explored the coding sequence evolution of the three gene pairs using various methods (summarized in Table 1, detailed in Supplemental File 1, and visualized using JalView [63] and manually annotated in Supplemental Figures 2, 3, and 4). The branch-site test for positive selection [47] of the PAML package [48] detected positive selection in the parental copies of Sep2. In this case the estimated value of $\omega$ was high, and two amino acid sites were identified by the Bayes Empirical Bayes [49] approach implemented in PAML. Sequence analysis by fixed effects likelihood method (FEL) [52] reveals that the majority of sites in each gene pair have evolved under negative selection. Analysis by mixed effects model of evolution (MEME) [53] detected a few sites under episodic diversifying selection in two of the gene pairs; however, only one instance of episodic diversifying selection, in Sep2 and Sep5 near the C-terminus, corresponds to an amino acid difference between the parent and retrogene. So, it appears that the majority of amino acid sites in all three gene pairs evolved under negative selection, indicating constraint on gene function, while positive selection does not appear to be a major factor influencing functional diversification in any of the three gene pairs presented. The multiple 


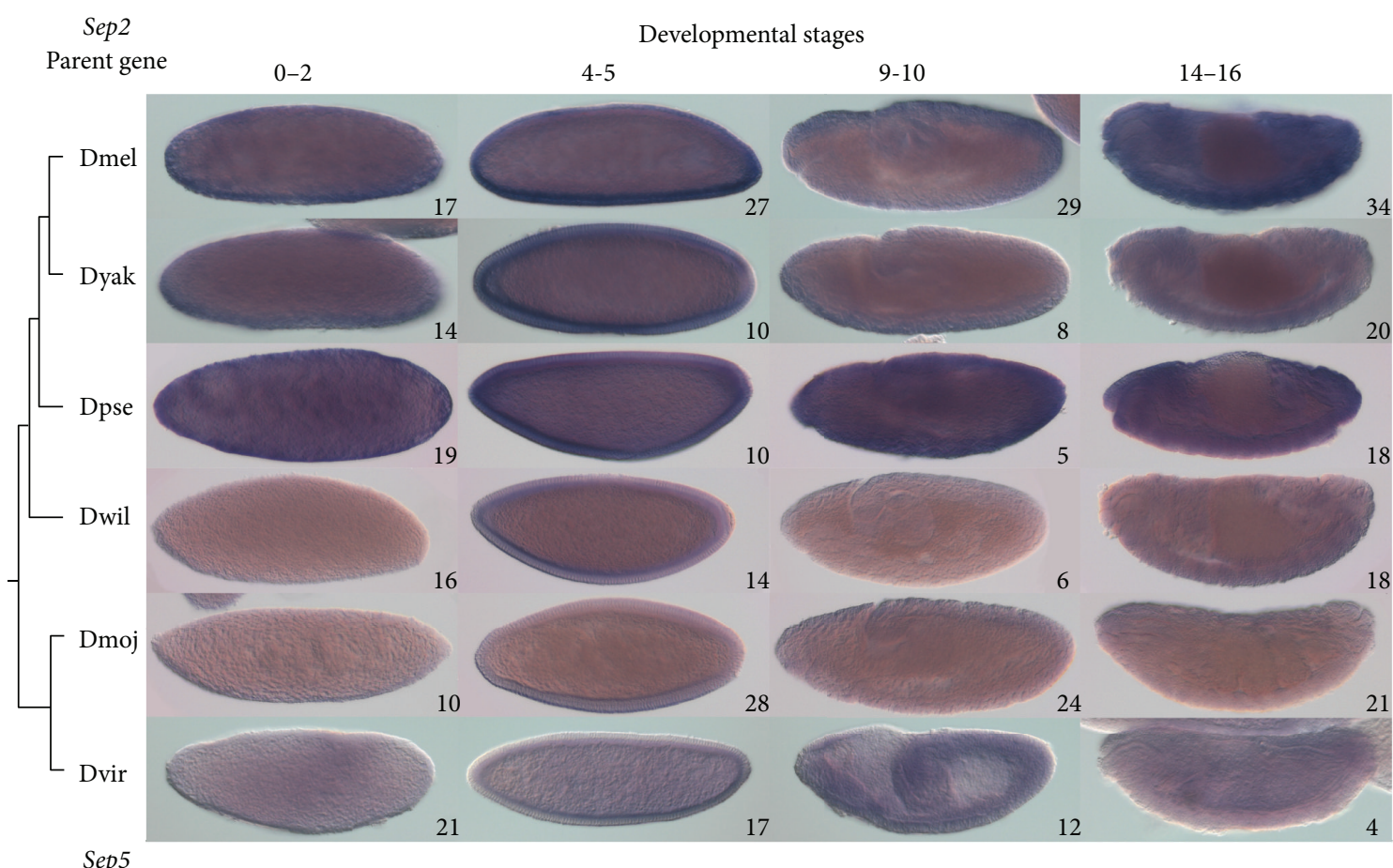

Retrogene

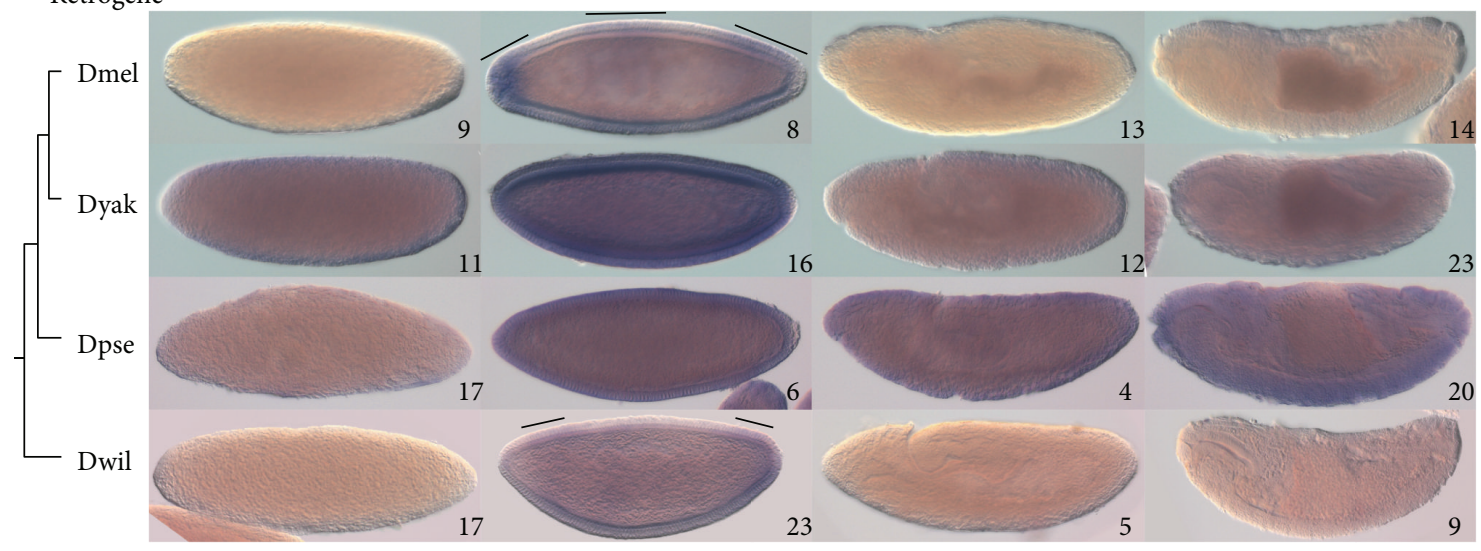

FIgURE 5: Expression patterns of Sep2 and Sep5. Expression of Sep2 and Sep5 was detected in Drosophila species by in situ hybridization as shown in the top and bottom panels, respectively. Purple coloration indicates presence of mRNA. Cladograms show the relationships of the species investigated. Numbers on individual panels indicate the number of embryos assessed with consistent staining patterns. Sep 5 showed domains of more intense staining during cellular blastoderm stages in D. melanogaster and D. willistoni; these domains are indicated by black bars. Stage ranges are according to [24]: 0-2, syncytial blastoderm; 3-4, cellular blastoderm; 9-10, during germ-band elongation; 14-16, final embryonic stages. Staining during stages 0-2 indicates presence of maternal transcripts [25]. Dmel, D. melanogaster; Dyak, D. yakuba; Dpse, D. pseudoobscura; Dwil, D. willistoni; Dmoj, D. mojavensis; Dvir, D. virilis.

sequence alignments of the gene pairs reveal several amino acid sites that are conserved among orthologs, but differ between paralogs. Sep2 and Sep5, which are both classified as group $1 \mathrm{~B}$ septins [64], show many of these amino acid sites, including differences in the G1 and G3 GTPase domains, the Sep1 motif, and within the coiled-coil domains (Supplemental Figure 4). These likely represent nonsynonymous substitutions that occurred early during the evolution of the three retrogenes, which were then maintained by negative selection.

\section{Conclusion}

Here we have determined embryonic expression patterns and performed sequence analyses to reveal some of the complexities in three cases of retroduplication. Our approach of comparing multiple species with and without a particular retrogene allowed us to investigate how the expression patterns of the gene pairs evolved in relation to one another and whether orthologs evolve similarly across species, unlike studies that only consider differences in transcript level across tissues 
TABLE 1: Sequence evolution analyses on three gene pairs.

\begin{tabular}{|c|c|c|c|c|c|c|c|c|c|}
\hline \multirow{3}{*}{ Gene pair } & \multicolumn{3}{|c|}{ Branch-site test (PAML) } & \multicolumn{4}{|c|}{ Datamonkey } & & \\
\hline & \multirow{2}{*}{$\begin{array}{l}\text { Total no. } \\
\text { of sites in } \\
\text { codon } \\
\text { alignment }\end{array}$} & \multirow{2}{*}{$\begin{array}{l}\text { Parent as } \\
\text { foreground } \\
\text { No. of } \\
\text { sites }^{\mathrm{a}}\end{array}$} & \multirow{2}{*}{$\begin{array}{l}\text { Retrogene as } \\
\text { foreground } \\
\text { No. of sites }\end{array}$} & \multicolumn{2}{|c|}{$\begin{array}{l}\text { Negatively selected } \\
\text { sites (FEL) }\end{array}$} & \multicolumn{2}{|c|}{$\begin{array}{l}\text { Sites under episodic } \\
\text { diversifying selection } \\
(\mathrm{MEME})\end{array}$} & \multicolumn{2}{|c|}{ Visually identified sites } \\
\hline & & & & $\begin{array}{l}\text { No. of } \\
\text { sites }^{\text {b }}\end{array}$ & $\begin{array}{l}\text { Proportion of } \\
\text { total sites }\end{array}$ & $\begin{array}{l}\text { No. of } \\
\text { sites }^{c}\end{array}$ & $\begin{array}{l}\text { Proportion } \\
\text { of total sites }\end{array}$ & $\begin{array}{l}\text { No. of } \\
\text { sites }^{\mathrm{d}}\end{array}$ & $\begin{array}{l}\text { Proportion } \\
\text { of total sites }\end{array}$ \\
\hline $\begin{array}{l}\text { CG8331 and } \\
\text { CG4960 }\end{array}$ & 155 & N/A & N/A & 95 & 0.613 & 4 & 0.026 & 2 & 0.013 \\
\hline $\begin{array}{l}\text { CG17734 and } \\
\text { CG11825 }\end{array}$ & 94 & N/A & N/A & 67 & 0.713 & 0 & 0 & 5 & 0.053 \\
\hline Sep2 and Sep5 & 398 & 2 & N/A & 356 & 0.894 & 3 & 0.0075 & 58 & 0.146 \\
\hline
\end{tabular}

${ }^{a}$ Number of positively selected sites identified by Bayes Empirical Bayes when the null model was rejected; ${ }^{\mathrm{b}}$ number of negatively selected sites with $P$ value $\leq$ 0.05 ; ${ }^{c}$ number of sites under episodic diversifying selection with $P$ value $\leq 0.05$; ${ }^{\mathrm{d}}$ number of sites with $\geq 80 \%$ amino acid conservation in the parent, $\geq 80 \%$ amino acid conservation in the retrogene, but which were different between parent and retrogene.

within a single species $[4,5,65,66]$. Our results are consistent with the concept that, because retroduplication separates the coding sequence of a parent gene from its transcriptional regulatory elements, retrogenes undergo diversification in expression pattern more readily compared to their parents $[3,66]$. The expression and sequence evolution of Sep2 and Sep5 seem to reflect Ohno's idea that one gene copy would maintain the ancestral function, allowing the other copy to diverge in function [67]. However, parent gene expression patterns do not have to remain static through evolution, as shown for the CG17734 and CG11825 gene pair.

\section{Acknowledgments}

The authors thank the Microscopy and Microanalysis Facility at UNB for the help with microscopy. This work was supported by a Natural Sciences and Engineering Research Council of Canada Discovery grant to Denise Clark.

\section{References}

[1] H. Kaessmann, N. Vinckenbosch, and M. Long, "RNAbased gene duplication: mechanistic and evolutionary insights," Nature Reviews Genetics, vol. 10, no. 1, pp. 19-31, 2009.

[2] J. Brosius, "Retroposons: seeds of evolution," Science, vol. 251, no. 4995, article 753, 1991.

[3] V. Katju, "In with the old, in with the new: the promiscuity of the duplication process engenders diverse pathways for novel gene creation," International Journal of Evolutionary Biology, vol. 2012, Article ID 341932, 24 pages, 2012.

[4] Y. Bai, C. Casola, C. Feschotte, and E. Betrán, "Comparative genomics reveals a constant rate of origination and convergent acquisition of functional retrogenes in Drosophila," Genome Biology, vol. 8, no. 1, article R11, 2007.

[5] M. G. I. Langille and D. V. Clark, "Parent genes of retrotransposition-generated gene duplicates in Drosophila melanogaster have distinct expression profiles," Genomics, vol. 90, no. 3, pp. 334-343, 2007.

[6] Y. Bai, C. Casola, and E. Betrán, "Evolutionary origin of regulatory regions of retrogenes in Drosophila," BMC Genomics, vol. 9, article 241, 2008.
[7] Y. Bai, C. Casola, and E. Betrán, "Quality of regulatory elements in Drosophila retrogenes," Genomics, vol. 93, no. 1, pp. 83-89, 2009.

[8] A. Force, M. Lynch, F. B. Pickett, A. Amores, Y. L. Yan, and J. Postlethwait, "Preservation of duplicate genes by complementary, degenerative mutations," Genetics, vol. 151, no. 4, pp. 1531$1545,1999$.

[9] E. Betrán, K. Thornton, and M. Long, "Retroposed new genes out of the X in Drosophila," Genome Research, vol. 12, no. 12, pp. 1854-1859, 2002.

[10] M. D. Vibranovski, Y. Zhang, and M. Long, "General gene movement off the X chromosome in the Drosophila genus," Genome Research, vol. 19, no. 5, pp. 897-903, 2009.

[11] R. P. Meisel, M. V. Han, and M. W. Hahn, "A complex suite of forces drives gene traffic from Drosophila X chromosomes," Genome Biology and Evolution, vol. 1, pp. 176-188, 2009.

[12] M. D. Vibranovski, H. F. Lopes, T. L. Karr, and M. Long, "Stagespecific expression profiling of Drosophila spermatogenesis suggests that meiotic sex chromosome inactivation drives genomic relocation of testis-expressed genes," PLoS Genetics, vol. 5, no. 11, Article ID e1000731, 2009.

[13] R. M. Waterhouse, E. M. Zdobnov, F. Tegenfeldt, J. Li, and E. V. Kriventseva, "OrthoDB: the hierarchical catalog of eukaryotic orthologs in 2011," Nucleic Acids Research, vol. 39, no. 1, pp. D283-D288, 2011.

[14] M. Calero, G. R. Whittaker, and R. N. Collins, "Yoplp, the yeast homolog of the polyposis locus protein 1, interacts with Yiplp and negatively regulates cell growth," Journal of Biological Chemistry, vol. 276, no. 15, pp. 12100-12112, 2001.

[15] A. Y. Gracey, J. V. Troll, and G. N. Somero, "Hypoxia-induced gene expression profiling in the euryoxic fish Gillichthys mirabilis," Proceedings of the National Academy of Sciences of the United States of America, vol. 98, no. 4, pp. 1993-1998, 2001.

[16] J. Wang, Y. Cao, Y. Chen, Y. Chen, P. Gardner, and D. F. Steiner, "Pancreatic $\beta$ cells lack a low glucose and $\mathrm{O}_{2}$-inducible mitochondrial protein that augments cell survival," Proceedings of the National Academy of Sciences of the United States of America, vol. 103, no. 28, pp. 10636-10641, 2006.

[17] L. H. Hartwell, J. Culotti, and B. Reid, "Genetic control of the cell-division cycle in yeast. I. Detection of mutants," Proceedings of the National Academy of Sciences of the United States of America, vol. 66, no. 2, pp. 352-359, 1970. 
[18] J. Saarikangas and Y. Barral, "The emerging functions of septins in metazoans," EMBO Reports, vol. 12, no. 11, pp. 1118-1126, 2011.

[19] J. C. Adam, J. R. Pringle, and M. Peifer, "Evidence for functional differentiation among Drosophila septins in cytokinesis and cellularization," Molecular Biology of the Cell, vol. 11, no. 9, pp. 3123-3135, 2000.

[20] K. Tamura, S. Subramanian, and S. Kumar, "Temporal patterns of fruit fly (Drosophila) evolution revealed by mutation clocks," Molecular Biology and Evolution, vol. 21, no. 1, pp. 36-44, 2004.

[21] A. G. Clark, M. B. Eisen, D. R. Smith et al., "Evolution of genes and genomes on the Drosophila phylogeny," Nature, vol. 450, no. 7167, pp. 203-218, 2007.

[22] D. L. Des Marais and M. D. Rausher, "Escape from adaptive conflict after duplication in an anthocyanin pathway gene," Nature, vol. 454, no. 7205, pp. 762-765, 2008.

[23] Y. Yang, Z. C. Hou, Y. H. Qian, H. Kang, and Q. T. Zeng, "Increasing the data size to accurately reconstruct the phylogenetic relationships between nine subgroups of the Drosophila melanogaster species group (Drosophilaidae, Diptera)," Molecular Phylogenetics and Evolution, vol. 62, no. 1, pp. 214-223, 2012.

[24] J. A. Campos-Ortega and V. Hartenstein, The Embryonic Development of Drosophila Melanogaster, Springer, 1997.

[25] D. K. Pritchard and G. Schubiger, "Activation of transcription in Drosophila embryos is a gradual process mediated by the nucleocytoplasmic ratio," Genes and Development, vol. 10, no. 9, pp. 1131-1142, 1996.

[26] S. Richards, Y. Liu, B. R. Bettencourt et al., "Comparative genome sequencing of Drosophila pseudoobscura: chromosomal, gene, and cis-element evolution," Genome Research, vol. 15, no. 1, pp. 1-18, 2005.

[27] S. E. Celniker, D. A. Wheeler, B. Kronmiller et al., "Finishing a whole-genome shotgun: release 3 of the Drosophila melanogaster euchromatic genome sequence," Genome Biology, vol. 3, no. 12, 2002.

[28] S. Tweedie, M. Ashburner, K. Falls et al., "FlyBase: enhancing Drosophila gene ontology annotations," Nucleic Acids Research, vol. 37, no. 1, pp. D555-D559, 2009.

[29] P. McQuilton, S. E. St Pierre, and J. Thurmond, "FlyBase 101: the basics of navigating FlyBase," Nucleic Acid Research, vol. 40, pp. 706-714, 2012.

[30] S. B. Needleman and C. D. Wunsch, "A general method applicable to the search for similarities in the amino acid sequence of two proteins," Journal of Molecular Biology, vol. 48, no. 3, pp. 443-453, 1970.

[31] S. F. Altschul, W. Gish, W. Miller, E. W. Myers, and D. J. Lipman, "Basic local alignment search tool," Journal of Molecular Biology, vol. 215, no. 3, pp. 403-410, 1990.

[32] E. Birney, M. Clamp, and R. Durbin, "GeneWise and Genomewise," Genome Research, vol. 14, no. 5, pp. 988-995, 2004.

[33] F. Sievers, A. Wilm, D. Dineen et al., "Fast, scalable generation of high-quality protein multiple sequence alignments using Clustal Omega," Molecular Systems Biology, vol. 7, article 539, 2011.

[34] M. Suyama, D. Torrents, and P. Bork, "PAL2NAL: robust conversion of protein sequence alignments into the corresponding codon alignments," Nucleic Acids Research, vol. 34, pp. W609W612, 2006.

[35] S. L. Baldauf, "Phylogeny for the faint of heart: a tutorial," Trends in Genetics, vol. 19, no. 6, pp. 345-351, 2003.

[36] K. Tamura, D. Peterson, N. Peterson, G. Stecher, M. Nei, and S. Kumar, "MEGA5: molecular evolutionary genetics analysis using maximum likelihood, evolutionary distance, and maximum parsimony methods," Molecular Biology and Evolution, vol. 28, no. 10, pp. 2731-2739, 2011.

[37] I. Letunic and P. Bork, "Interactive tree of life (iTOL): an online tool for phylogenetic tree display and annotation," Bioinformatics, vol. 23, no. 1, pp. 127-128, 2007.

[38] I. Letunic and P. Bork, "Interactive Tree of Life v2: online annotation and display of phylogenetic trees made easy," Nucleic Acids Research, vol. 39, no. 2, pp. W475-W478, 2011.

[39] S. Rozen and H. Skaletsky, "Primer3 on the WWW for general users and for biologist programmers," Methods in Molecular Biology, vol. 132, pp. 365-386, 2000.

[40] J. M. Ruijter, C. Ramakers, W. M. H. Hoogaars et al., "Amplification efficiency: linking baseline and bias in the analysis of quantitative PCR data," Nucleic Acids Research, vol. 37, no. 6, article e45, 2009.

[41] M. Kubista and R. Sindelka, "The prime technique: real-time PCR data," G.I.T. Laboratory Journal, vol. 9-10, pp. 33-35, 2007.

[42] D. Tautz and C. Pheifle, "A non-radioactive in situ hybridization method for the localization of specific RNAs in Drosophila embryos reveals translational control of the segmentation gene hunchback," Chromosoma, vol. 98, no. 2, pp. 81-85, 1989.

[43] R. Weiszmann, A. S. Hammonds, and S. E. Celniker, "Determination of gene expression patterns using high-throughput RNA in situ hybridization to whole-mount Drosophila embryos," Nature Protocols, vol. 4, no. 5, pp. 605-618, 2009.

[44] E. Lécuyer, N. Parthasarathy, and H. M. Krause, "Fluorescent in situ hybridization protocols in Drosophila embryos and tissues," Methods in Molecular Biology, vol. 420, pp. 289-302, 2008.

[45] A. Krogh, B. Larsson, G. Von Heijne, and E. L. L. Sonnhammer, "Predicting transmembrane protein topology with a hidden Markov model: application to complete genomes," Journal of Molecular Biology, vol. 305, no. 3, pp. 567-580, 2001.

[46] J. Trigg, K. Gutwin, A. E. Keating, and B. Berger, "Multicoil2: predicting coiled coils and their oligomerization states from sequence in the twilight zone," PLoS One, vol. 6, no. 8, Article ID e23519, 2011.

[47] J. Zhang, R. Nielsen, and Z. Yang, "Evaluation of an improved branch-site likelihood method for detecting positive selection at the molecular level," Molecular Biology and Evolution, vol. 22, no. 12, pp. 2472-2479, 2005.

[48] Z. Yang, "PAML 4: phylogenetic analysis by maximum likelihood," Molecular Biology and Evolution, vol. 24, no. 8, pp. 15861591, 2007.

[49] Z. Yang, W. S. W. Wong, and R. Nielsen, "Bayes empirical Bayes inference of amino acid sites under positive selection," Molecular Biology and Evolution, vol. 22, no. 4, pp. 1107-1118, 2005.

[50] W. Delport, A. F. Y. Poon, S. D. W. Frost, and S. L. Kosakovsky Pond, "Datamonkey 2010: a suite of phylogenetic analysis tools for evolutionary biology," Bioinformatics, vol. 26, no. 19, Article ID btq429, pp. 2455-2457, 2010.

[51] S. L. Kosakovsky Pond and S. D. W. Frost, "Datamonkey: rapid detection of selective pressure on individual sites of codon alignments," Bioinformatics, vol. 21, no. 10, pp. 2531-2533, 2005.

[52] S. L. Kosakovsky Pond and S. D. W. Frost, "Not so different after all: a comparison of methods for detecting amino acid sites under selection," Molecular Biology and Evolution, vol. 22, no. 5, pp. 1208-1222, 2005.

[53] B. Murrell, J. O. Wertheim, S. Moola, T. Weighill, K. Scheffler, and S. L. Kosakovsky Pond, "Detecting individual sites subject 
to episodic diversifying selection," PLOS Genetics, vol. 8, no. 7, Article ID e100276, 2012.

[54] P. Tomancak, B. P. Berman, A. Beaton et al., "Global analysis of patterns of gene expression during Drosophila embryogenesis," Genome Biology, vol. 8, no. 7, article R145, 2007.

[55] P. Tomancak, A. Beaton, R. Weiszmann et al., "Systematic determination of patterns of gene expression during Drosophila embryogenesis," Genome Biology, vol. 3, no. 12, 2002.

[56] V. R. Chintapalli, J. Wang, and J. A. T. Dow, "Using FlyAtlas to identify better Drosophila melanogaster models of human disease," Nature Genetics, vol. 39, no. 6, pp. 715-720, 2007.

[57] B. R. Graveley, A. N. Brooks, J. W. Carlson et al., "The developmental transcriptome of Drosophila melanogaster," Nature, vol. 471, no. 7339, pp. 473-479, 2011.

[58] Y. Zhang, D. Sturgill, M. Parisi, S. Kumar, and B. Oliver, "Constraint and turnover in sex-biased gene expression in the genus Drosophila," Nature, vol. 450, no. 7167, pp. 233-237, 2007.

[59] N. Vinckenbosch, I. Dupanloup, and H. Kaessmann, "Evolutionary fate of retroposed gene copies in the human genome," Proceedings of the National Academy of Sciences of the United States of America, vol. 103, no. 9, pp. 3220-3225, 2006.

[60] J. Wang, M. Long, and M. D. Vibranovski, "Retrogenes moved out of the $\mathrm{z}$ chromosome in the silkworm," Journal of Molecular Evolution, vol. 74, no. 3-4, pp. 113-126, 2012.

[61] C. M. Field, O. Al-Awar, J. Rosenblatt, M. L. Wong, B. Alberts, and T. J. Mitchison, "A purified Drosophila septin complex forms filaments and exhibits GTPase activity," Journal of Cell Biology, vol. 133, no. 3, pp. 605-616, 1996.

[62] K. G. Guruharsha, J. F. Rual, B. Zhai et al., "A protein complex network of Drosophila melanogaster," Cell, vol. 147, no. 3, pp. 690-703, 2011.

[63] A. M. Waterhouse, J. B. Procter, D. M. A. Martin, M. Clamp, and G. J. Barton, "Jalview Version 2-A multiple sequence alignment editor and analysis workbench," Bioinformatics, vol. 25, no. 9, pp. 1189-1191, 2009.

[64] F. Pan, R. L. Malmberg, and M. Momany, "Analysis of septins across kingdoms reveals orthology and new motifs," $B M C$ Evolutionary Biology, vol. 7, article 103, 2007.

[65] A. C. Marques, I. Dupanloup, N. Vinckenbosch, A. Reymond, and H. Kaessmann, "Emergence of young human genes after a burst of retroposition in primates," PLoS Biology, vol. 3, no. 11, article e357, 2005.

[66] B. P. Cusack and K. H. Wolfe, "Not born equal: increased rate asymmetry in relocated and retrotransposed rodent gene duplicates," Molecular Biology and Evolution, vol. 24, no. 3, pp. 679-686, 2007.

[67] S. Ohno, Evolution By Gene Duplication, Springer, 1970. 

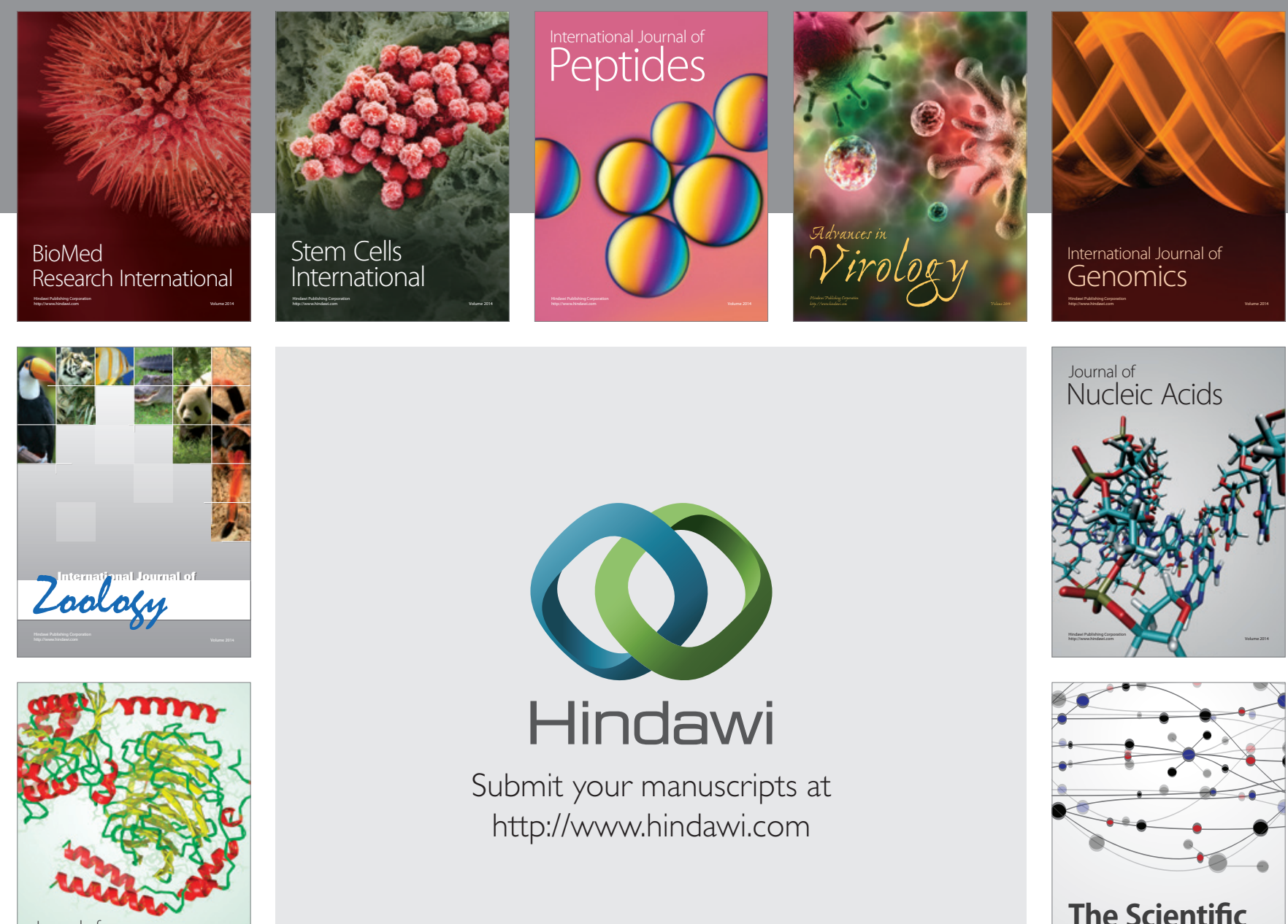

Submit your manuscripts at

http://www.hindawi.com

Journal of
Signal Transduction
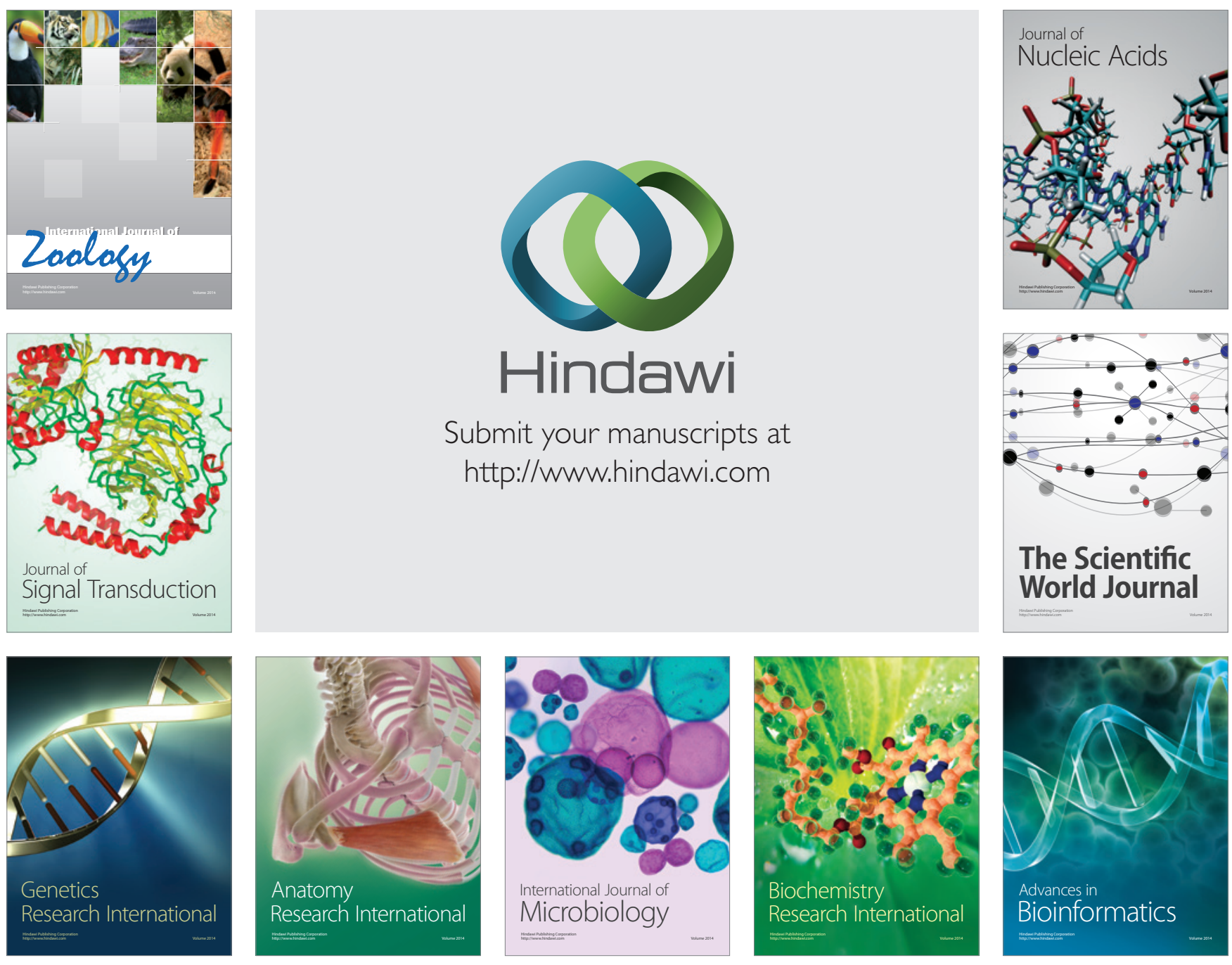

The Scientific World Journal
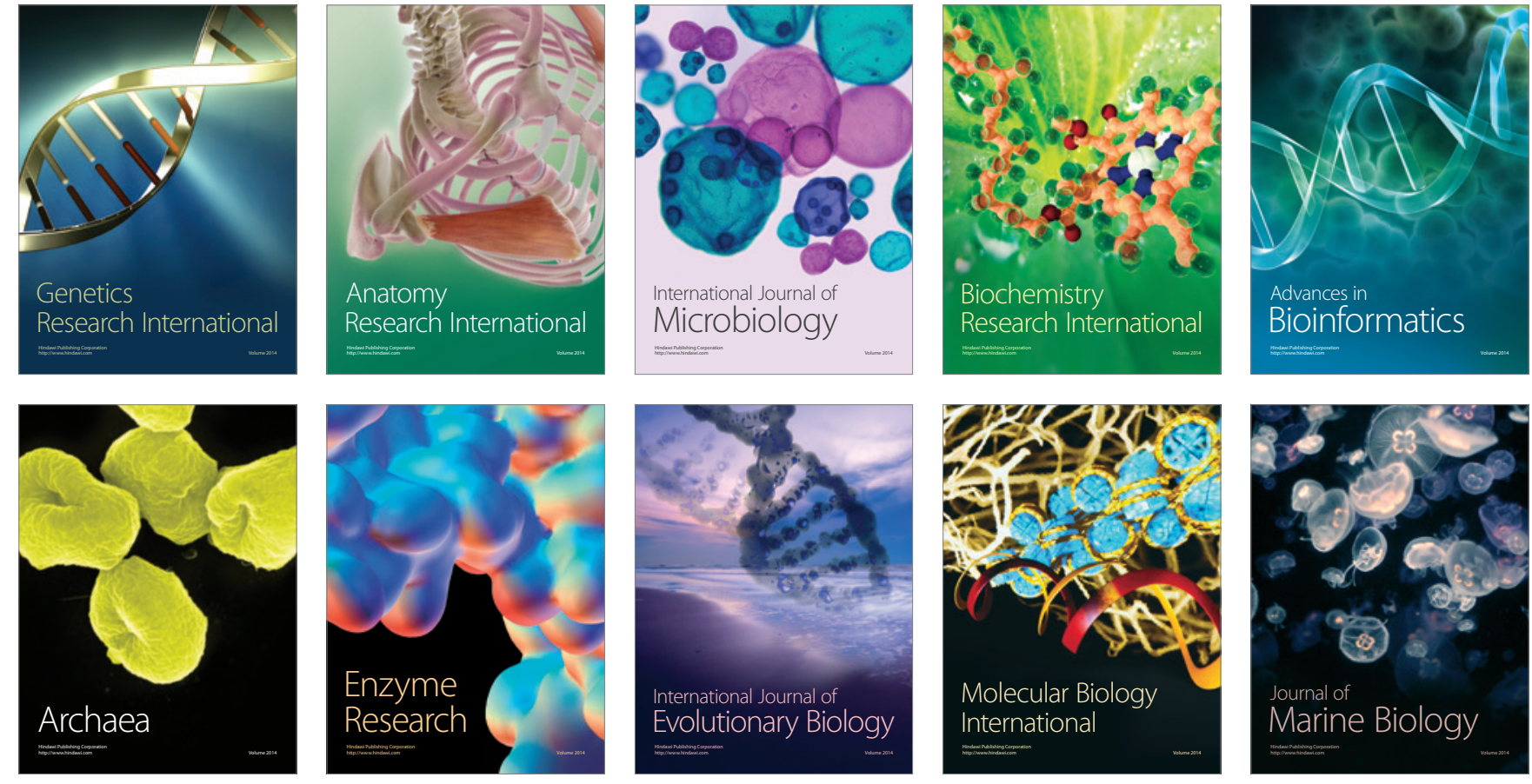